\title{
Colliding Crystalline Beams
}

\author{
A. F. Haffmans, D. Maletic, and A. G. Ruggiero \\ Brookhaven National Laboratory, Upton, NY 11973, USA
}

Crystalline Beams ${ }^{*}$ are an ordered state of an ensemble of ions, circulating in a storage ring, with very small velocity fluctuations $[1,2]$. They can be obtained from ordinary warm ion beams with the application of powerful cooling techniques (stochastic, electron, laser, ...). Depending on the focussing properties and dimensions of the storage ring, and on the ion beam density, several ground states are possible [3-4]. All of them can be visualized as a bundle of $n_{s}$ symmetrically distributed, parallel strings [5]. The longitudinal ion separation $\lambda$ is the same for all strings. The minimum temperature that can be achieved depends on the background noise of the cooling technique used. It is required for stability that the vibration amplitude of the ions is only a fraction of the separation $\lambda$.

\section{COLLIDING CRYSTALLINE BEAMS}

There are several advantages in using colliding Crystalline Beams. First, it is possible to obtain more-compact beams. Second, most importantly, the particles in each beam occupy well-defined and rigid positions. The uncertainty on the transverse location of each string is considerably smaller than the longitudinal spacing $\lambda$. By carefully aligning the two beams to pair countermoving strings in the collision, it is possible to increase the luminosity by a considerable factor. Indeed the luminosity in this case is proportional to the number of strings in each beam

$$
\mathbf{L}=\mathrm{n}_{\mathrm{s}} \mathrm{L}_{\mathrm{s}}
$$

where

$$
\mathrm{L}_{\mathrm{s}}=\mathrm{N}_{\mathrm{s}}^{2} \mathrm{f}_{\mathrm{enc}} / 4 \pi \sigma_{\mathrm{eff}}^{2}
$$

is the contribution to the luminosity from each pair of countermoving strings; $\mathrm{f}_{\text {enc }}$ is the frequency of encounter, $\sigma_{\text {eff }}$ is an effective cross section which is the common transverse dimension of the two strings during collision, and $\mathrm{N}_{s}$ is the number of circulating ions per string engaged in the collision at one time, assuming that the two countermoving beams are identical.

\section{COLLISION BETWEEN STRINGS}

The easiest example to investigate is the case of two identical Crystalline Beams sharing the same storage ring. The two beams are moving in the opposite direction along the common closed orbit. We require that the ions in the two beams have the same magnetic rigidity, mass number $A$, and charge state $Q$. But they must have opposite sign of the electric charge, if the

* Work performed under the auspices of the US DOE bending along the closed orbit is due to a common axial magnetic field. Thus, one beam is made of partially-stripped, positively-charged ions, and the other beam is made of the same ions with an excess of electrons (negative ions). We shall not debate here how this can be realized in practice, but only assume that it is possible.

It is also sufficient to investigate the case of collision between paired strings and neglect the presence of the others in the background which are taken to be at a sufficiently large distance. We shall assume that the two countermoving strings are initially separated vertically by some distance $2 \mathrm{~b}$, and want to investigate the behavior and the stability of the motion of the ions when the separation distance $b$ is reduced and the strings are brought closer for collision.

\section{EQUATIONS OF MOTION}

If the two strings were moving in the same direction, the configuration expected is that of a vertical zigzag [4] of which the properties are known. In the present case, the two strings move parallel to each other, but in opposite direction, each with velocity $\beta c$. As the ions of one string pass along the reference ion in the other string, the motion of this is modulated periodically in all directions. We shall assume that the modulation has an amplitude small compared to the separation $\lambda$, and linearize the equations of motion which apply, for symmetry, to all particles. They are [5]:

$$
\begin{aligned}
& b^{\prime \prime}+\mathrm{K}_{\mathrm{v}}(\mathrm{s}) b+\mathrm{K}_{\mathrm{sc}} \mathrm{f}_{3}(\alpha, \tau) b / \mathrm{g}_{0}=0 \\
& \mathrm{a}^{\prime \prime}+\mathrm{K}_{\mathrm{h}}(\mathrm{s}) \mathrm{a}=\mathrm{h}(\mathrm{s}) \delta \\
& \sigma^{\prime}=\mathrm{h}(\mathrm{s}) \mathrm{a}-\delta / \gamma^{2} \\
& \delta^{\prime}=-\left(\gamma^{2} \lambda \mathrm{K}_{\mathrm{sc}} / 2 \mathrm{~g}_{0}\right)\left[\mathrm{df}_{1}(\alpha, \tau) / \mathrm{d} \tau\right]
\end{aligned}
$$

The force acting on the test particle is the one generated by all the ions of the countermoving string. Those ions on the same string do not contribute to the total force. In Eq.s (3-6) the independent variable is the path length $s$ along the common closed orbit. A prime denotes differentiation with respect to $\mathrm{s}$. $\mathrm{b}$ is the vertical displacement from the median plane of symmetry, $a$ is the radial displacement, $\sigma$ the path length difference, and $\delta$ the momentum deviation from the reference closed orbit conditions. $\mathrm{K}_{\mathrm{v}, \mathrm{h}}$ are the focussing functions which we approximate with constants $\left(v_{v, h} / R\right)^{2}$, where $v_{v, h}$ are the betatron tune numbers and $R$ is the average closed orbit radius. $h(s)$ is the curvature function, which we also approximate with $1 / R$. $\beta$ and $\gamma$ are respectively the velocity and energy relativistic factors. $g_{0}=1.3$ is a constant. The space-charge constant [5] 


\section{DISCLAIMER}

This report was prepared as an account of work sponsored by an agency of the United States Government. Neither the United States Government nor any agency thereof, nor any of their employees, make any warranty, express or implied, or assumes any legal liability or responsibility for the accuracy, completeness, or usefulness of any information, apparatus, product, or process disclosed, or represents that its use would not infringe privately owned rights. Reference herein to any specific commercial product, process, or service by trade name, trademark, manufacturer, or otherwise does not necessarily constitute or imply its endorsement, recommendation, or favoring by the United States Government or any agency thereof. The views and opinions of authors expressed herein do not necessarily state or reflect those of the United States Government or any agency thereof. 


\section{DISCLAIMER}

Portions of this document may be illegible in electronic image products. Images are produced from the best available original document. 


$$
K_{s c}=\left(2 / R^{2}\right)\left(\lambda_{c} / \lambda\right)^{3}
$$

where the critical spacing [5]

$$
\lambda_{c}=\left(g_{0} Q^{2} r_{0} R^{2} / A \beta^{2} \gamma^{5}\right)^{1 / 3}
$$

and $\mathrm{r}_{0}=1.535 \times 10^{-18} \mathrm{~m}$ is the proton classical radius. Finally, the form factors

$$
f_{n}(\alpha, \tau)=\Sigma_{i}\left[\alpha^{2}+(i-\tau)^{2}\right]^{-n / 2}
$$

where the aspect ratios $\alpha=2 b / \lambda \gamma$ and $\tau=2 \mathrm{~s} / \lambda$. The form factors are periodic with respect to $\tau$ with a periodicity of one.

\section{CASE OF LARGE SEPARATION}

In the limit of large separation among the two strings, that is $\alpha>1, f_{3}$ is about constant, whereas $\mathrm{df}_{1} / d \tau$ oscillates around zero. In the smooth and linear approximation, ignoring the curvature of the storage ring, we can decouple the vertical motion from the longitudinal one. Because the two beams have opposite charge sign and $f_{3}>0$ for all values of $\alpha$ and $\tau$, it is seen that the third term at the 1.h. side of Eq. (3) adds to the focussing of the storage ring. We can estimate the betatron tune-shifit as

$$
\delta v_{v}=K_{s c}<f_{3}>R^{2} / 2 v_{0} g_{0}
$$

where $\left\langle\mathrm{f}_{3}\right\rangle \sim 2 / \alpha^{2}$. If the tune-shift does not exceed half of the distance $\Delta v_{\mathrm{v}}$ to the nearest half-integral stopband the motion is stable [5].

At the same time, particles oscillate longitudinally $(\sigma)$ around their stationary position with an amplitude

$$
\sigma_{0} / \lambda=\lambda^{2} \mathrm{~K}_{\mathrm{sc}} \Delta / 8 \mathrm{~g}_{0}
$$

where $\Delta<0.01$ for $\alpha>1$ and is a slow function of $\alpha$. Thus, for large separation between the countermoving strings, the motion is stable, and the configuration of each string in not disrupted.

\section{CASE OF CLOSE COLLISION}

As the two strings are brought together in collision, we reach a range of aspect ratios $\alpha<1$. In this range, the form factor $f_{3}$ essentially vanishes over the full period of $\tau$, except that periodically, at $\tau=$ integer, it transforms to a kick. In the smooth approximation, we can spread the kick evenly over one period. Solving Eq. (3) in the same approximation again leads to the tune-shift (10). Since $\left\langle\mathrm{f}_{3}\right\rangle \sim 2 / \alpha^{2}$, Eq. (10) sets a limit to the aspect ratio $\alpha$ below which the relation cannot be satisfied. At the limit of stability of individual strings, $\lambda \sim \lambda_{c}$, there is a threshold value of the aspect ratio

$$
\alpha^{2}>1 / v_{v} \Delta v_{v}=\alpha_{1 \text { rans }}^{2}
$$

below which the configuration of the two strings is destroyed. As a consequence, a large betatron tune $v_{v}$ and a large periodicity of the storage ring is desired (so that $\Delta v_{v} \sim v_{v} / 2$ ) to allow the lowest possible threshold value $\alpha_{\text {trans }}$.

As the separation $2 b$ between the two strings reduces, the longitudinal force acting on the test particle has still an oscillatory behavior around zero, but with a waveform grossly distorted from a pure sine-wave. The amplitude of the oscillation increases as $\Delta \sim 1 / 2 \alpha^{2}$ for $\alpha \ll 1$. Inserting this at the r.h. side of Eq. (11) gives the amplitude of the longitudinal oscillations. To avoid disruption of both strings, we require $\sigma_{0}<\lambda / 2$. That is, at the limit of individual string stability, when $\lambda-\lambda_{c}$, the aspect ratio cannot be reduced below the threshold value

$$
\alpha^{2}>(\lambda / R)^{2} / 4 \mathrm{~g}_{0}=\alpha_{\text {long }}{ }^{2}
$$

\section{CONCLUSIONS}

In conclusion, two strings, moving in the opposite direction in the same storage ring, separated vertically by the aspect ratio $\alpha$, have a stable configuration with the ions performing smallamplitude oscillations around their othervise stationary position, provided that the vertical separation is sufficiently large. Two criteria are to be satisfied. namely Eq.s (12 and 13). In a typical storage ring, it is found that the limitation is caused mainly by the transverse forces, and that the smallest separation that can be achieved between the two strings is set by the betatron tune

$$
\alpha=2 b / \lambda \gamma-1 / v_{v}
$$

We have seen that according to Eq. (2) the luminosity depends on the effective cross section $\sigma_{\text {eff }}$ of the two strings colliding with each other, given by the background noise of the cooling method used. For the effective collision of the two strings, it is also required that the separation b, set by Eq.s (12 or 13 ), is sufficiently smaller than $\sigma_{\text {eff }}$.

\section{REFERENCES}

[1] A. Rahman and J. P. Schiffer, Phys. Rev. Lett., 57, (1986), 1133.

[2] R. W. Hasse and J. P. Schiffer, Ann. of Phys., 203, (1990), 419.

[3] J. Wei, X. P. Li and A. M. Sessler, Phys. Rev. Lett., 73, (1994), 3089.

[4] A. F. Hatfmans, D. Maletic, and A. G. Ruggiero, "Crystalline Beams ...", Internal Reports BNL-60436/60619/60743/ 60876 (1994).

[5] A. G. Ruggiero, "Crystalline Beams: Theory, Experiments, and Proposals", Internal Report BNL-61007 (1994) 